\title{
Diagnosis, phenomenology and treatment of poststroke depression
}

\section{Diagnóstico, fenomenologia e tratamento da depressão pós-acidente vascular cerebral (AVC)}

\author{
Sergio E Starkstein ${ }^{\mathrm{a}}$ and Alicia Lischinsky ${ }^{\mathrm{b}}$ \\ ${ }^{a}$ Institute of Neuroscience, University of Buenos Aires, Buenos Aires Neuropsychiatric Center, and Salto Municipal Hospital. ${ }^{b}$ Raul Carrea Institute \\ of Neurological Research. Buenos Aires, Argentina
}

\begin{abstract}
Diagnosing depression in stroke patients is a challenge in neuropsychiatry since depression symptoms may overlap neurological deficit signs. The best approach is to assess the presence of depressive symptoms using semi-structured or structured psychiatric interviews, such as the Present State Exam, the Structured Clinical Interview for DSM-IV or the Schedules for Clinical Assessment in Neuropsychiatry. The diagnosis of a depressive syndrome should be made according to standardized diagnostic criteria for mood disorders due to neurological disease such as in the DSM-IV or ICD-10. Depression rating scales such as the Hamilton Depression Scale and the Center for Epidemiologic Scales for Depression may be used to rate the depression severity and monitor the progression of antidepressant treatment. Most studies have reported the effectiveness of pharmacological treatment in patients with post-stroke depression, and there is preliminary evidence that the degree of impairment in activities of daily living (ADL) may improve as well.
\end{abstract}

Keywords Depression. Stroke. Anxiety. Antidepressants.

Resumo O diagnóstico de depressão em pacientes pós-acidente vascular cerebral (AVC) representa um desafio para a neuropsiquiatria visto que os sintomas de depressão podem se sobrepor aos sinais de déficit neurológico. A melhor abordagem é avaliar a presença de sintomas depressivos fazendo uso de entrevistas psiquiátricas estruturadas ou semi-estruturadas, como a Entrevista Psiquiátrica Polivalente Padronizada (Present State Exam), a Entrevista Clínica Estruturada para DSM-IV (Structured Clinical Interview for DSM-IV) ou os Quadros para Avaliação Clínica em Neuropsiquiatria (Schedules for Clinical Assessment in Neuropsychiatry). O diagnóstico de uma síndrome depressiva deve ser feito segundo os critérios diagnósticos padronizados para transtornos do humor devido a doença neurológica como no DSM-IV ou ICD-10. As escalas de classificação da depressão, como a Escala de Avaliação da Depressão de Hamilton (Hamilton Depression Scale) e Centro de Escalas Epidemiológicas para Depressão (Center for Epidemiologic Scales for Depression), podem ser usadas para classificar a gravidade da depressão e monitorar a evolução do tratamento antidepressivo. Na maioria dos estudos, relata-se a eficácia do tratamento medicamentoso em pacientes com depressão pós-AVC. Existem evidências preliminares de que o grau de comprometimento das atividades diárias pode também ser reduzido.

Descritores Depressão. Ataque. Ansiedade. Antidepressivos

\section{Introduction}

Depression has been recognized as a frequent complication of stroke lesions. The prevalence of depression among stroke patients admitted to rehabilitation centers was reported to range between $49 \%$ and $54 \%$, whereas community-based studies reported a range between $18 \%$ and $23 \%$.
Depression was reported to negatively affect recovery from impairments in daily living activities, and is also associated with higher mortality. Since post-stroke depression can be adequately treated with antidepressant drugs, it is important to make a reliable and valid diagnosis of depression in order to start early treatment. 
This present article reviews the main phenomenological aspects of post-stroke depression, standardized diagnostic criteria to diagnose this disorder, the most useful structured psychiatric interviews and depression rating scales to assess the presence and severity of depression after stroke, and the main pharmacological treatments available.

\section{Strategies to diagnosing depression in patients with neurological disorders}

Cohen-Cole and Stoudemire ${ }^{1}$ suggested four different strategies of diagnosing depression in physically ill patients: 1) the "inclusive" strategy, which counts depressive diagnostic symptoms whether or not they may be related to physical illness; 2) the "etiological" strategy in which symptoms are counted only if the examiner considers they are not a result of the disease itself; ${ }^{2} 3$ ) the "substitutive" strategy in which autonomic symptoms of depression are replaced by psychological symptoms; ${ }^{3}$ and 4) the "exclusive" strategy in which symptoms are removed from the diagnostic criteria whenever they are no more frequently seen in depressed vs. non-depressed patients. $^{4}$

The relative usefulness of these strategies to diagnose depression in stroke patients was studied by Paradiso et $\mathrm{al}^{5}$ who assessed a series of 142 patients with an acute stroke lesion who were followed-up at 3, 6, 12 and 24 months after stroke. Throughout the follow-up period, those patients reporting a depressed mood during the acute stroke hospitalization $(n=60,42 \%)$ showed a significantly higher frequency of autonomic and psychological symptoms of depression compared to the group who did not have in-hospital depressed mood, except for symptoms of early morning awakening, loss of libido, weight loss, suicide ideas, and pathological guilt.

Paradiso et al also calculated the frequency of patients meeting the DSM-IV ${ }^{6}$ criteria for major depression using some of the different diagnostic strategies described above. It was found that frequencies of major depression at the initial evaluation were $18 \%$ for the inclusive strategy (i.e., depressive symptoms were counted regardless of their presumed cause), and $22 \%$ for the substitutive approach (i.e., all autonomic symptoms were eliminated, and major depression was diagnosed based on the presence of at least four psychological symptoms plus depressed mood). Another important finding was that three autonomic symptoms (autonomic anxiety, morning depression, and subjective anergy) were significantly more frequent in stroke patients with in-hospital depressed mood as compared to patients with no depressed mood at all times throughout the 2-year follow-up period. Moreover, the specificity of unmodified DSM-IV criteria had a sensitivity of $100 \%$ and a specificity that ranged from 95 to $98 \%$ as compared to criteria that only included specific symptoms. Based on these findings, Robinson" suggested that "modifying DSM-IV criteria because of the existence of an acute medical illness is probably unnecessary".

In conclusion, different strategies can be used to diagnose depression in stroke patients. Few studies examined the reliability and validity of depressive symptoms for the diagnosis of depression in this population, but most of them showed a high concordance of autonomic and affective symptoms of depression, as well as their consistency over time.

\section{Diagnostic criteria for post-stroke depression}

One of the main dilemmas in the field of neuropsychiatry is how to diagnose mood disorders such as depression and anxiety in patients with neurological illnesses when symptoms of the mood disorder may be produced by the neurological disease itself. For instance, insomnia, psychomotor retardation, loss of appetite, loss of libido, and loss of energy can be expected findings in patients with stroke lesions or traumatic brain injuries, but also constitute cardinal symptoms for the diagnosis of depression among patients with "primary" (i.e., no known brain injury) depression.

Standardized diagnostic criteria for depression in neurological disease can be found in both the Diagnostic and Statistical Manual for Mental Disorders, $4^{\text {th }}$ edition (DSM-IV), and the International Classification of Diseases, $10^{\text {th }}$ edition (ICD-10). ${ }^{8}$ The DSM-IV includes the category of "Mood Disorder due to a General Medical Condition" (Table 1). This category consists of 2 subtypes: one with depressive features, whenever the predominant mood is depressed but the full criteria for a major depressive episode are not met, and another with major depressive-like episodes, whenever the full criteria for a major depressive episode are met. The ICD-10 (Diagnostic Criteria for Research) includes a section of mental disorders due to brain damage and dysfunction and to physical disease (Table 2). If these criteria are met, mood disorders can be diagnosed whenever the criteria for specific affective disorders are also met. The DSM-IV defines post stroke major depression as "a mood disorder due to stroke with major depressive-like episode," and also provides research criteria for minor depression, which requires depression or anhedonia with at least one but less than four additional symptoms of major depression.

The diagnosis of depression in neurological illness should be made after a thorough mental status examination in which the specific symptoms of affective disorders are systematically assessed. Ideally, the presence of depressive symptoms should

Table 1 - Diagnostic criteria for a Mood disorder Due to a General Medical Condition (adapted from DSM-IV)

\footnotetext{
A. A prominent and persistent disturbance in mood predominates in the clinical picture and is characterized by either (or both) of the following:

(1) Depressed mood or markedly diminished interest or pleasure in all, or almost all, activities

(2) Elevated, expansive, or irritable mood

B. There is evidence from the history, physical examination, or laboratory findings that the disturbance is the direct physiological consequence of a general medical condition

C. The disturbance is not better accounted for by another mental disorder.

D. The disturbance does not occur exclusively during the course of a delirium.

E. The symptoms cause clinically significant distress or impairment in social, occupational, or other important areas of functioning.
} 
Table 2 - Other mental disorders due to brain damage and dysfunction and to physical disease (adapted from ICD-10)

G1. There is objective evidence (from physical and neurological examination and laboratory tests) and/or history of cerebral disease, damage, or dysfunction, or of systemic physical disorder known to cause cerebral dysfunction, including hormonal disturbances (other than alcohol or other psychoactive substance-related) and non-psychoactive drug effects.

G2. There is a presumed relationship between the development (or marked exacerbation) of the underlying disease, damage, or dysfunction, and the mental disorder.

G3. There is recovery from or significant improvement in the mental disorder following removal or improvement of the underlying presumed cause.

G4. There is insufficient evidence for an alternative causation of the mental disorder, e.g., a strong family history or a clinically similar or related disorder. If criteria G1, G2, and G4 are met, a provisional diagnosis is justified; if, in addition, there is evidence of G3, the diagnosis can be regarded as certain.

be assessed with a semi-structured or structured psychiatric interview, such as the Present State Exam (PSE). ${ }^{9}$ This interview consists of a "probe and question" structure allowing both examiner and subject to develop, interpret, and elaborate upon inquires about symptoms with a glossary of symptom definition. The Schedules for Clinical Assessment in Neuropsychiatry (SCAN) is a set of instruments that assess, measure, and classify the major psychiatric disorders of adult life. ${ }^{10}$ The SCAN includes the full PSE-10, an Item Group Checklist (IGC), which enables the rating of information obtained from case-records or informants other than the proband, and a Clinical History Schedule, filled out with information obtained from the proband, informants, and clinical records. The SCAN allows the use of different algorithms to generate diagnoses, such as in ICD-10 and DSM-IV. This instrument should be used by trained psychiatrists who attended a specific course at a World Health Organization training center.

The Structured Clinical Interview for DSM-IV (SCID) is a semi-structured interview for making the major Axis I and Axis II diagnoses. ${ }^{11}$ After the usual mental status evaluation, a clinician can use specific sections of the SCID to confirm a DSMIV diagnosis. Alternatively, the whole SCID can be assessed with the purpose of diagnosing all Axis I or II disorders present in the patient. For those patients with a known neurological disorder it is better to use the Non-Patient edition (SCID-NP), which includes an overview section to assess history of psychopathology. It is important to stress that the examiner should use all sources of information available at the time of the evaluation (e.g., notes, reports from relatives or caregivers, etc.). The examiner is also asked to use her/his own judgment to determine whether a specific symptom is present or not. The SCID usually takes from 60 to 90 minutes to be completed.

Depression rating scales are useful to rate the severity of depressive disorders. They can also be used as screening instruments to determine the likelihood of a given psychiatric diagnosis. However, as Robinson suggested, the "gold standard" for diagnosing depression is standardized diagnostic criteria obtained during the clinical interview. Among the several widely used depression scales are the Hamilton Depression Scale (HAM-D), the Beck Depression Inventory (BDI), ${ }^{12}$ the Zung Depression Scale (ZDS),${ }^{13}$ the General Health Questionnaire (GHQ), ${ }^{14}$ the Montgomery-Asberg Depression Rating Scale, ${ }^{15}$ and the Center for Epidemiological Scales for Depression (CES-D). ${ }^{16}$ The HAM-D is a 17 -item interviewerrated scale that measures psychological and autonomic symptoms of depression. This scale assesses, for instance, the individual's interest in daily life activity and work productivity; however, rating these elements in a patient with a neurological disorder requires some judgment. Other HAM-D items, such as sleep problems, psychomotor retardation, loss of appetite, weight loss, lack of energy, loss of libido, and hypochondria can also be difficult to rate in patients with neurological conditions.

In conclusion, the diagnosis of depression in neurological disease is still a complicated issue. Both the DSM-IV and the ICD-10 include specific categories to diagnose depression in patients with neurological disease (e.g., "Mood Disorder due to a General Mood Condition" in DSM-IV, and "Organic Affective Disorder" in ICD-10). Several investigators have described syndromes of major depression, dysthymia, and minor depression in patients with either stroke lesions or traumatic brain injury. Further studies are necessary to examine the validity of these syndromal constructs.

\section{Phenomenology of post-stroke depression}

It is still a matter of discussion whether the overlap and potential non-specificity of depressive symptoms in stroke patients require specific assessment and diagnostic strategies. Lipsey et $\mathrm{a} \mathrm{l}^{17}$ compared the frequency of depressive symptoms between a series of 43 patients with post-stroke major depression and a group of 43 age-comparable patients with primary depression. Both groups showed a similar profile of depressive symptoms, such as simple depression, general anxiety, affective flattening, self-neglect, tension, lack of energy, worrying, and irritability. The only symptom's clusters that were significantly different were slowness (more frequent in stroke patients), and loss of interest and concentration (more frequently seen in primary depression patients). Based on these findings, Lipsey et al suggested that the clinical symptoms of major depression following stroke are similar to those found in elderly depressed patients without brain damage.

Fedoroff et $\mathrm{al}^{18}$ assessed depressive symptoms in a consecutive series of 205 patients with acute stroke, who were divided into those who reported a depressed mood (41\%) and those who reported no mood change (59\%). Both groups were comparable on main demographic variables such as gender, socioeconomic status, marital status, and personal or family history of psychiatric disorders. In contrast, patients who reported a depressed mood were younger and had significantly more severe cognitive and functional impairments than patients without a depressed mood. Patients with depressed mood had a significantly higher frequency of autonomic and psycho- 
logical symptoms of depression, except for early morning awakening. Patients with depressed mood had an average of 4 autonomic and 4 psychological symptoms of depression as compared to an average of 1 autonomic and 1 psychological symptom of depression in patients without a depressed mood. Based on additional findings, Fedoroff et al estimated that the use of standardized diagnostic criteria (such as the DSM-III) might inaccurately increase the frequency of major depression by $1 \%$ to $2 \%$.

Fedoroff et al also assessed the percentage of patients who failed to meet the diagnostic criteria for major depression due to denial of a depressed mood but met all the required diagnostic criteria for major depression. It was found that only $5 \%$ of the sample met the above conditions (i.e., meeting all necessary symptoms for major depression except depressed mood). If these patients were included with the ones with major depression who acknowledged a depressed mood, the frequency of major depression would have increased from $24 \%$ to $29 \%$. Hence, Fedoroff et al concluded that both autonomic and psychological symptoms of depression were significantly related to the presence of a depressed mood among patients with acute stroke.

\section{Pharmacological treatment of post-stroke depression}

Lipsey and coworkers ${ }^{19}$ conducted the first controlled treatment trial of post-stroke depression and studied 39 stroke patients who met the diagnostic criteria for either major or minor depressive disorder. Patients were randomly assign to receive nortriptyline $(\mathrm{NTP})(\mathrm{n}=17)$ or placebo $(n=22)$. Five patients in the NTP group dropped out of the study: one complained of dizziness, one of sedation, two developed episodes of delirium, and one patient had a sudden syncopal episode of unknown etiology after four weeks of treatment. In the placebo group there were seven drop outs: one patient developed mania, one refused to be further interviewed after week 1 , one died of severe congestive heart failure, one complained of dizziness, two patients were discharged from hospital before the study was completed and was lost to follow up, and one patient died of intracerebral hemorrhage. The major finding of study was that patients on NTP had significantly greater mood improvement than the placebo group as ascertained by a greater than $50 \%$ reduction in Ham-D scores and a final Ham-D ${ }^{20}$ score below 10 points. All 11 patients who completed the course of NTP treatment were responders, as compared to only five of the 15 placebo patients $(p=0.001)$. There was no significant difference between the NTP and placebo groups in terms of either improvement in cognitive function (as measured with the Mini Mental State Examination), or deficits in activities of daily living.

The second double-blind treatment study carried out in poststroke depression patients included 27 patients with or without evidence of depression, who were approximately 6 weeks post-stroke (Reding et $\mathrm{al}^{21}$ ). All patients were randomly assigned to treatment with either trazodone hydrochloride or placebo. Trazodone was started at a nightly dose of $50 \mathrm{mg}$ and was increased by $50 \mathrm{mg}$ every three days up to a total nightly dose of $200 \mathrm{mg}$. The dose was either decreased or maintained for an additional of 3 days whenever the evaluating physician noted possible adverse side effects. It was found that patients with clinical diagnosis of depression who were on trazodone had a greater improvement in their Barthel activities of daily living score as compared to the placebo group. One limitation of the study was that mood improvement was not part of the outcome measures. Also, it could not be determined whether trazodone produced a significantly greater mood improvement as compared to placebo. Main side effects of trazodone were sedation and eye discomfort. Six patients receiving placebo dropped out of the study because of perceived side effects ( 4 with sedation and 1 complaining of eye discomfort) and one patient refused to continue in the study for unspecified reasons.

Andersen et $\mathrm{al}^{22}$ carried out a 6 -week double-blind, randomized, placebo-controlled study in a series of 66 stroke patients with a Ham-D score $>13$ points. Thirty-three patients were randomized to citalopram and 33 patients to placebo. Citalopram was started at a dose of $20 \mathrm{mg} /$ day or $10 \mathrm{mg}$ /day in patients over 66 years old. There were seven dropouts in the citalopram group: 1 died of an aortic aneurysm, 1 suffered a new stroke, 1 developed a skin rash, 1 violated the protocol, 1 complained of dizziness, 1 developed anxiety, and 1 complained of headaches. Two patients dropped out of the placebo group: 1 died of cardiac failure and 1 patient developed dizziness. Results of efficacy analyses demonstrated a significantly better outcome with citalopram than placebo at both 3 and 6 weeks using either the Ham-D or the Melancholia Scale as outcome measures. Side effects of citalopram where mostly mild and included nausea, vomiting, and fatigability.

Gonzalez-Tordecillas et $\mathrm{al}^{2}$ carried out a 6-week open-label treatment trial of fluoxetine and nortriptyline in stroke patients with depression. The study included 26 patients treated with fluoxetine ( $20 \mathrm{mg}$ in the morning), 11 patients were treated with nortriptyline $(25 \mathrm{mg} /$ day increased over 9 days to $75 \mathrm{mg} /$ day, then adjusted according to plasma level), and 11 depressed patients were given no treatment. Although fluoxetine and nortriptyline showed similar efficacy, both antidepressants were superior to placebo as measured by HAM-D scores, a scale of neurological impairment, the Barthel index, and $\mathrm{MMSE}^{24}$ scores. Only three patients dropped out of the study and no serious adverse effects occurred.

Dam et $\mathrm{al}^{25}$ examined the efficacy of fluoxetine and maprotiline on functional recovery in post-stroke hemiplegic patients undergoing rehabilitation therapy. Fifty-two severely disabled hemiplegic subjects were randomly assigned to 3 treatment groups during a 3-month physical therapy: mapotriline $(150 \mathrm{mg} / \mathrm{d})$, fluoxetine $(20 \mathrm{mg} / \mathrm{d})$, or placebo. The main finding was that rehabilitation itself or combined with either antidepressant produced significant gains in the Barthel index and neurological deficit improvement. The greatest improvements were observed in the fluoxetine-treated patients, and fluoxetine was superior to physical therapy alone. Both fluoxetine and mapotriline also produced a significant mood improvement as compared to placebo. Side effects of mapotriline included epileptic seizures (1 patient), and seda- 
tion (2 patients). Two patients dropped out of the fluoxetine group because of seizures, and 2 patients reported transient nausea and insomnia.

Dahmen $^{26}$ examined the efficacy of venlafaxine in an openlabel treatment study that included 12 patients with stroke lesions. Venlafaxine treatment was associated with a significant reduction in depression scores and significant improvements in scores measuring neurological deficits. The drug was well tolerated in all patients. Side effects were mild and included elevated liver enzymes (1 patient), agitation (1 patient), and nausea (3 patients).

Wiart et $\mathrm{al}^{27}$ carried-out a multicenter, double-blind, placebo-controlled study using fluoxetine in patients with a recent single ischemic or hemorrhagic stroke. Thirty-one of 121 patients screened were included in a 6-week study: 16 received $20 \mathrm{mg} / \mathrm{d}$ of fluoxetine and 15 received placebo. The main finding was that fluoxetine-treated patients had a significantly greater decrease in depression scores as compared to the placebo group.

Palomaki et $\mathrm{al}^{28}$ examined the prophylactic antidepressive efficacy of mianserin in a randomized, double-blind trial that included 100 consecutive patients with an acute ischemic stroke. Patients received $60 \mathrm{mg}$ /day of mianserin $(\mathrm{n}=51)$ or placebo $(n=49)$ for a year. Only 5 patients had major depression at the initial evaluation, and no significant differences occurred between treatment groups regarding frequencies of major depression at any time point during the follow-up period.

In a recent double-blind placebo-controlled study, Robinson et $\mathrm{al}^{29}$ compared nortriptyline, fluoxetine and placebo in the treatment of depression and in recovery from physical and cognitive impairments after stroke. One-hundred and four patients with acute stroke, with or without depression, were included in the study. There were 21 patients who dropped out of the study: 9 dropouts were from the depressed fluoxetine group (3 patients complained of gastrointestinal symptoms and 6 refused further treatment), and 3 belonged to the depressed nortriptyline group ( 2 patients had medical deterioration and 1 patient refused further treatment). Nortriptyline was found to be significantly more effective than fluoxetine and placebo in the treatment of both depression and anxiety symptoms, and in improving recovery in activities of daily living. Contrasting, neither medication had a significant effect on cognitive functioning as measured by the MMSE, or on social functioning as measured by the Social Functioning Exam. Side effects in the fluoxetine group included anxiety, gastrointestinal symptoms, insomnia and weight loss, whereas the most frequent side effect in the nortriptyline group was sedation.

In summary, most studies published to date have reported adequate effectiveness of the pharmacological treatment in patients with post-stroke depression. The use of antidepressants in this group of patients was reported to produce a significant improvement of depressive symptoms. There is preliminary evidence that the degree of impairment in ADL may also improve.

\section{Conclusions}

Several studies demonstrated that stroke patients with depressed mood have a significantly higher prevalence of autonomic and psychological symptoms of depression when compared to stroke patients without a depressed mood. Poststroke depression patients were found to have a similar profile of autonomic and psychological symptoms of depression as compared to patients with primary or functional depression. Diagnosis of depression should be carried out with structured psychiatric interviews, such as the SCID or SCAN, and the severity of depression should be rated according to specific depression rating scales, such as the HAM-D. Depression can be diagnosed using the DSM-IV category of "Mood Disorder due to a General Medical Condition" or the ICD-10 criteria for "Mental Disorders due to Brain Damage and Dysfunction". Two randomized, double blind, placebo-controlled studies demonstrated a significantly higher efficacy of nortriptyline over placebo in stroke patients. Though selective serotonin reuptake inhibitors (SSRIs), such as citalopram, also showed to be adequately effective in poststroke depression, a recent study suggests that tricyclics may be more effective than SSRIs.

\section{Acknowledgments}

This study was partially supported by a grant from the Raúl Carrea Institute of Neurological Research-FLENI.

\section{References}

1. Cohen-Cole SA, Stoudemire A. Major depression and physical illness: special considerations in diagnosis and biologic treatment. Psychiatric Clin N Am 1987;10:1-14.

2. Rapp SR, Vrana S. Substituing nonsomatic for somatic symptoms in the diagnosis of depression in elderly male medical patients. Am J Psychiatry 1989;146:1197-200.

3. Endicott J. Measurement of depression in patients with cancer. Cancer 1984;53(suppl):2443-248

4. Bukberg J, Pennan D, Holland JC. Depression in hospitalized cancer patients. Psychosomatic Med 1984;46:99-212.

5. Paradiso S, Ohkubo T, Robinson RG. Vegetative and psychological symptoms associated with depressed mood over the first two years after stroke. Intern J Psychiatry Med 1997;27:137-57.

6. American Psychiatric Association. Diagnostic and statistical manual of mental disorders DSM-IV. Washington (DC): American Psychiatric Press; 1994.

7. Robinson RG. The clinical neuropsychiatry of stroke. Cambridge: Cambridge University Press; 1999.

8. World Health Organization. The ICD-10 classification of mental and behavioral disorders. Geneva; 1994

9. World Health Organization. Schedules for clinical assessment in neuropsychiatry. Version 2.0. Geneva; 1994.

10. Spitzer RL, Williams JB, Gibbon M, First MB. The structured clinical interview for DSM-III-R (SCID): I. History, rationale, and description. Arch Gen Psychiatry 1992;49:624-9. 
11. Beck AT, Ward CH, Mendelson M. An inventory for measuring depression. Arch Gen Psychiatry 1961;4:551-71.

12. Wing JK, Cooper JE, Sartorius N. Measurement and classification of psychiatric symptoms. Cambridge: Cambridge University Press; 1974.

13. Goldberg DP, Hiller VF. A scaled version of the general health questionnaire. Psychological Med 1979;9:139-98.

14. Montgomery SA, Asberg M. A new depression scale designed to be sensitive to change. Br J Psychiatry 1979;134:382-9.

15. Radloff LA. The CES-D Scale, a self-report depression scale for research in the general population. Appl Psychol Meas 1977;1:385-401.

16. Lipsey JR, Spencer WC, Rabins PV, Rabins PV, Robinson RG . Phenomenological comparison of functional and poststroke depression. Am J Psychiatry 1986;143:527-9.

17. Fedoroff JP, Starkstein SE, Parikh RM, Price TR, Robinson RG. Are depressive symptoms non-specific in patients with acute stroke? Am J Psychiatry 1991;148:1172-6.

18. Lipsey JR, Spencer WC, Rabins PV, Rabins PV, Robinson RG . Phenomenological comparison of functional and poststroke depression. Am J Psychiatry 1986;143:527-9.

19. Hamilton MA. A rating scale for depression. J Neurol Neurosurgery Psychiatry 1960;23:56-62.

20. Reding JJ, Orto LA, Winter SW, Fortuna IM, DiPonte P, McDowell FH. Antidepressant therapy after stroke: a double-blind trial. Arch Neurol 1986;43:763-5.

21. Andersen G, Vestergaard K, laruitzen L. Effective treatment of poststroke depression with the selective serotonin reuptake inhibitor citalopram. Stroke 1994;25:1099-104.

22. Gonzalez-Torrecillas JL, Hildebrand J, Medlewicz J, Lobo A. Effects of early treatment of post-stroke depression on neuropsychological rehabilitation. Int Psychogeriatrics 1995;78:547-60.

23. Folstein MF, Folstein SE, McHugh PR. Mini-Mental state: a practical method for grading the cognitive state of patients for the clinician. $\mathrm{J}$ Psychiat Res 1975;12:189-98.
24. Dam M, Tonin P, De Boni A, Pizzolato G, Casson S, Ermani M, et al. Effects of fluoxetine and maprotiline on functional recovery in poststroke hemiplegic patients undergoing rehabilitation therapy. Stroke 1996;27:1211-4.

25. Dahmen N. Therapy of early poststroke depression with venlafaxine: safety, tolerability, and efficacy as determined in a open, uncontrolled clinical trial. Stroke 1999;30:691-2.

26. Wiart L, Petit H, Joseph PA, Mazaux JM, Barat M. Fluoxetine in early poststroke depression. Stroke 2000;31:1829.

27. Palomäki H, Kaste M, Berg A, Lönnqvist J, Lehtihalmes M, Hares J. Prevention of poststroke depression: 1 year randomised placebo controlled double blind trial of mianserin with 6 month follow up after therapy. J Neurol Neurosurgery Psychiatry 1999;66:490-4.

28. Robinson RG, Schultz SK, Castillo C, Kopel T, Kosier JT, Newman RM, et al. Nortiptyline versus Fluoxetine in the treatment of depression and in short-term recovery after stroke: a placebo-controlled, doubleblind study. Am J Psychiatry 2000;157:351-9.

\section{Correspondência}

Sergio E. Starkstein

Buenos Aires Neuropsychiatric Center

Ciudad de la Paz 1808, $4^{\text {th }}$ floor

1428 Buenos Aires, Argentina

Tels.: (00xx54) (11) 4788-6960 e 4788-8206

E-mails: ses@ceneba.com.ar e guistar@infovia.com.ar 